\title{
Newly Onset Sinus Bradycardia in the Context of Multiple Sclerosis Relapse
}

\author{
Sandra Jurić ${ }^{1}$, Antonija Mišmaš ${ }^{2}$, Nina Mihić ${ }^{1}$, Ana Marija Barać ${ }^{3}$ and Mario Habek ${ }^{2,4}$
}

\begin{abstract}
Cardiovascular disorders in acute multiple sclerosis (MS) relapse have been infrequently reported. We present a young multiple sclerosis patient with acute onset of cerebellar symptomatology along with sinus bradycardia. Brain magnetic resonance imaging showed one lesion in the left cerebellar hemisphere which showed postcontrast enhancement and one in the midbrain without postcontrast enhancement. No cardiac pathology was found and symptoms gradually improved after a 5-day course of corticosteroid therapy. It is important to bear in mind the possibility of these rare cardiac symptoms in MS patients, because of their timely recognition and appropriate treatment.
\end{abstract}

Key words: multiple sclerosis, relapse, sinus bradycardia

(Intern Med 51: 1121-1124, 2012)

(DOI: 10.2169/internalmedicine.51.7139)

\section{Introduction}

Cardiovascular disorders in acute multiple sclerosis (MS) relapse have been infrequently reported, in contrast to other acute neurological diseases like subarachnoid hemorrhage, stroke, head trauma, tumors or cervical spine injury $(1,2)$. The aim of this case report was to describe an abnormal heart rhythm as one of the presenting symptoms of MS relapse.

\section{Case Report}

A 20-year-old woman was referred to neurological emergency service due to deterioration of the underlying disease (relapsing-remitting MS) in the form of impaired speech, vision blurring, clumsiness of left limbs and walking instability. The patient had been diagnosed with MS three years previously and was treated with beta interferon $1 \mathrm{~b}$ for two years. Since treatment was started she had two relapses, including this one. Neurological examination showed scanning speech, gaze-evoked rotational nystagmus, left limbs and gait ataxia. Her blood pressure was 110/65 $\mathrm{mmHg}$. She had no chest pain or breathlessness nor impending signs of syncope and her chest and heart auscultation revealed bradycardia, but no signs of cardiac murmurs, heart failure or pulmonary problems. There were no other symptoms or signs of autonomic dysfunction. Electrocardiogram (ECG) demonstrated heart frequency of 40 beats per minute (Fig. 1A).

Hematological and biochemical tests including troponin I and thyroid hormones were unremarkable. A cardiologist was consulted; treadmill showed an adequate increase in heart rate frequency and transthoracic echocardiography showed regular valve morphology and ventricular function with no sign of intracardiac masses or pericardial effusion. Except for bradycardia, holter ECG demonstrated findings within normal limits.

Brain magnetic resonance imaging (MRI) showed multiple periventricular demyelinating lesions and two infratentorial lesions, one in the left cerebellar hemisphere and one in the midbrain (Fig. 2, 3). Lesions in the left cerebellar hemisphere and in the right and left frontal centrum semiovale showed postcontrast enhancement.

Given that there was no cardiac pathology, it was concluded that the bradycardia was caused by a CNS lesion, in this case acute MS relapse, and a high dose of intravenous

\footnotetext{
${ }^{1}$ Department of Neurology, University Hospital Mostar, Bosnia and Herzegovina, ${ }^{2}$ Department of Neurology, Referral Center for Demyelinating Diseases of the Central Nervous System, University Hospital Center Zagreb, Croatia, ${ }^{3}$ Department of Family Medicine, Medical Faculty University of Mostar, Bosnia and Herzegovina and ${ }^{4}$ Department of Neurology, University of Zagreb, School of Medicine, Croatia Received for publication December 17, 2011; Accepted for publication January 23, 2012 Correspondence to Dr. Mario Habek, mhabek@mef.hr
} 


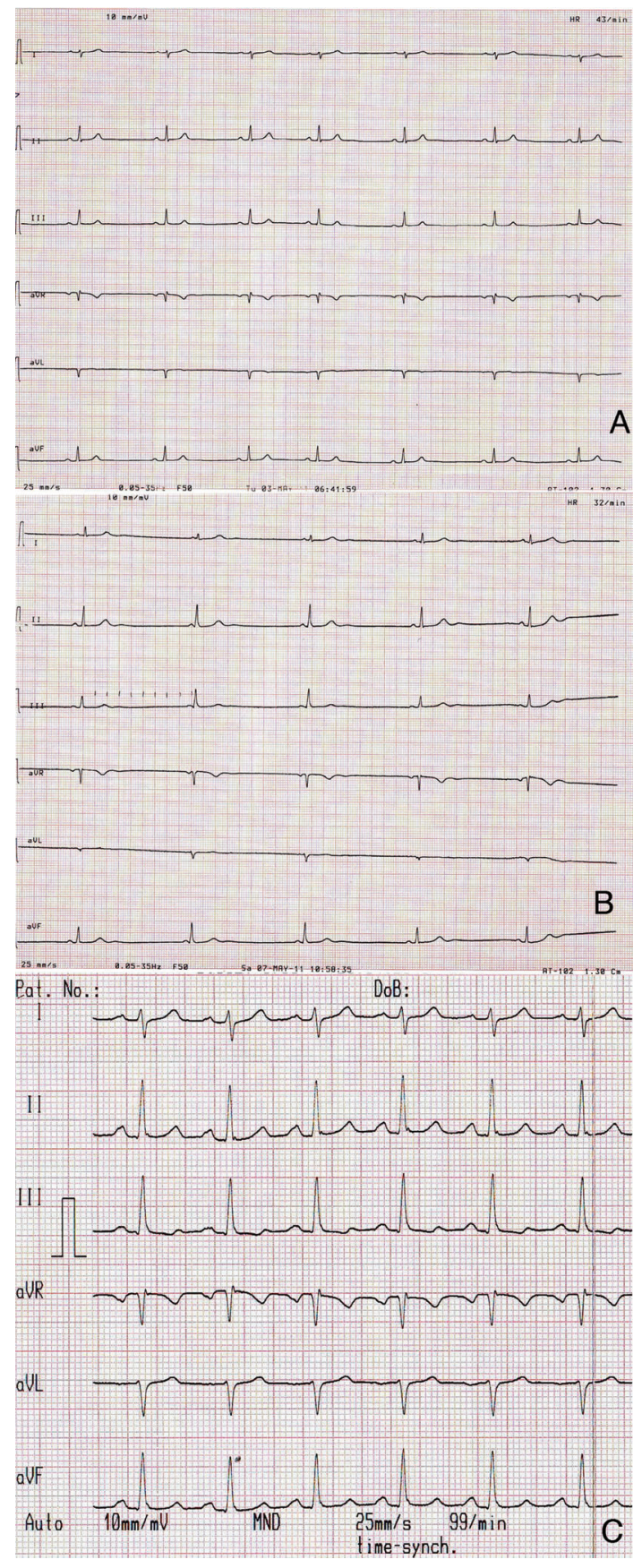

Figure 1. A) ECG on admission showing sinus bradycardia, 43/min; B) ECG on day 5 of corticosteroid therapy showing sinus bradycardia, 32/min; C) ECG a day following corticosteroid therapy showing normal sinus rhythm.

methylprednisolone (1 gram per day) was started for 5 days. On the last day of pulse corticosteroid therapy ECG showed even worse bradycardia (Fig. 1B). Even then the patient showed no signs of imminent syncopal episode and there were no other symptoms or signs of autonomic dysfunction.

She remained on continuous cardiac monitoring and one day after the last infusion of methylprednisolone, the frequency began to rise gradually over several days and there was no need for additional, more invasive therapy. The patient was discharged and fully recovered few days later, with heart frequency within normal limits (Fig. 1C) and no cere- bellar deficit whatsoever. After six months of follow-up there were no more episodes of bradycardia.

\section{Discussion}

Cardiovascular abnormalities in general, especially heart rhythm disturbances are rare presentations of MS relapse, unlike in other acute neurological conditions (3). It is believed that these arise from an excessive release of catecholamines acting on cardiac myocytes causing myonecrotic changes predominantly in subendocardial regions. Since the cardiac conduction system is also a subendocardial structure, some question the impact of these changes on cardiac conduction and rhythm (4).

There are few case reports describing cardiogenic shock, paroxysmal atrial fibrillation and neurogenic pulmonary edema associated with MS exacerbations (5-8). Some of the latter correlated with caudal brainstem lesions on MR imaging $(7,9)$. It was also reported that gliomas and vascular lesions in the medulla oblongata as well as surgical manipulations in this area were accompanied with bradycardia due to vagal involvement $(10,11)$. In one case report, neurocysticercosis of the right cerebellar peduncle was described as the underlying cause of bradycardia with the proposed mechanism of brainstem compression (12).

Some animal model studies indicate midbrain cuneiform nucleus and superior colliculus as important structures in cardiovascular changes. Cuneiform nucleus stimulation from the dorsomedial nucleus of the hypothalamus inhibited baroreflex bradycardia and noradrenalin microinjection into the superior colliculus evoked a dose-dependent blood pressure increase and heart rate decrease $(13,14)$. The main CNS regions involved in bradycardia are the dorsomedial nucleus of the hypothalamus and the dorsolateral periaqueductal grey matter which inhibit the cardiac baroreflex response and cause bradycardia. It has also been proposed that the midbrain cuneiform nucleus is the main link between these two areas (13). On the other hand, stimulation of sublobule IX-b of the posterior vermis of the cerebellum provokes a cardiovascular response characterized by hypotension and bradycardia, and an accompanying decrease of phrenic nerve activity (15).

The coincidence of sinus bradycardia with the appearance of other neurological deficits along with a good response to high-dose steroid therapy in the absence of underlying cardiac pathology, has led us to conclude that bradycardia was a symptom of MS relapse in the present patient. Nevertheless, it is difficult to conclude which lesion was the cause of sinus bradycardia. The most likely candidate is a midbrain lesion; however this lesion was not active. On the other hand in MS patients with clear brainstem clinical presentation, only $54 \%$ have brainstem MRI lesion that can explain the symptoms (16). As well, more widespread brainstem disfunction may not be visualised with MRI, but present on neurophysiological tests (17). Therefore, involvement of nerve fibers and the combination of the midbrain and cere- 

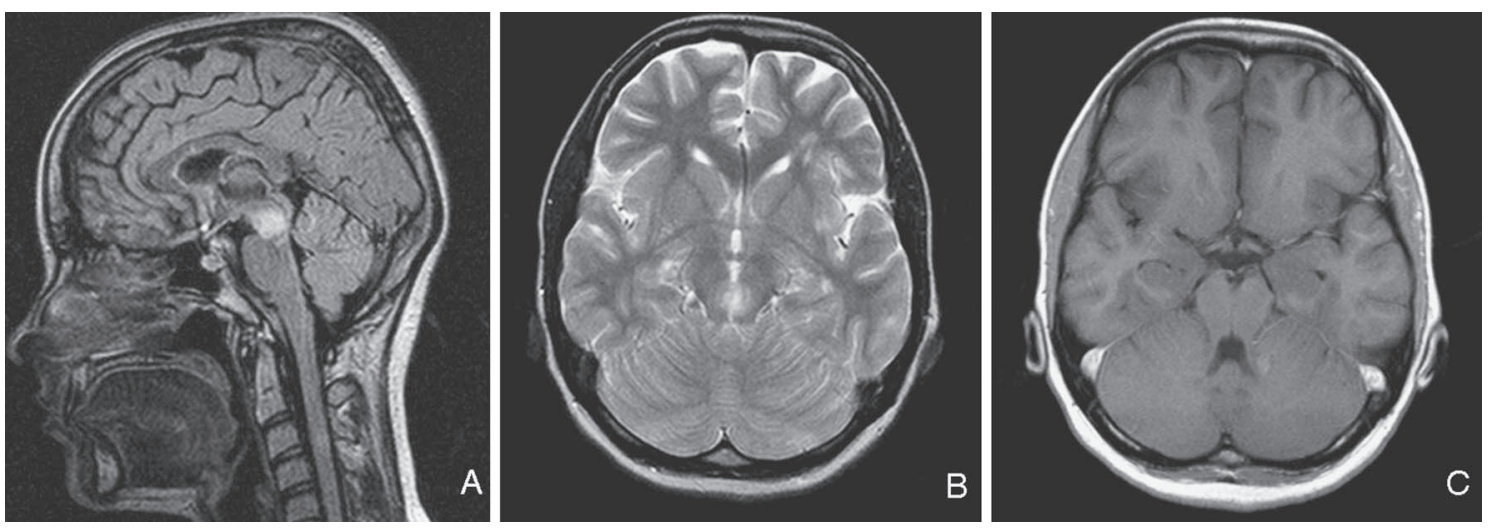

Figure 2. Brain MRI A) FLAIR saggital sequence; B) T2 transversal sequence; C) T1 postcontrast transversal sequence; showing two infratentorial lesions, one in the left cerebellar hemisphere and one in the midbrain with the lesions in the left cerebellar hemisphere showing postcontrast enhancement.

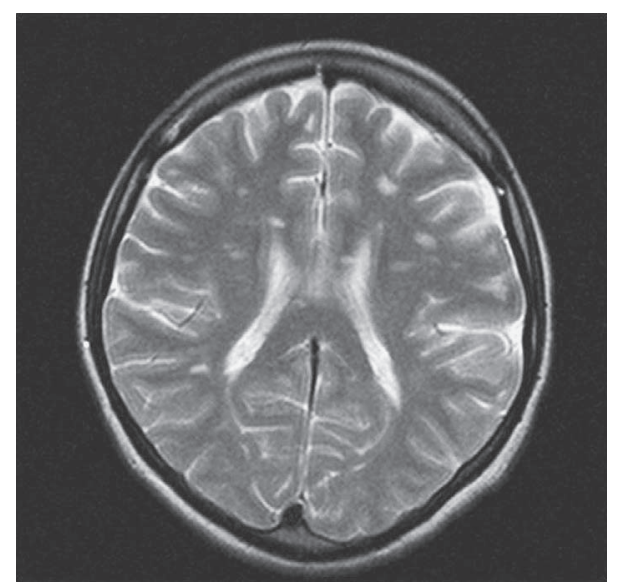

Figure 3. Brain MRI, T2 transversal sequence, showing multiple periventricular, subcortical and juxtacortical lesions typical for MS.

bellar lesions and their connections, probably resulted in bradycardia in the present patient. In conclusion it is important to bear in mind the possibility of these rare cardiac symptoms in MS patients, because of their timely recognition and appropriate treatment.

The authors state that they have no Conflict of Interest (COI).

Authors' contributions: Case report concept and design: Jurić, Habek and Mišmaš. Acquisition of data: Jurić and Mihić. Drafting of the manuscript: Jurić and Barać. Critical revision of the manuscript for important intellectual content: Jurić, Mišmaš, Mihić, Barać, Habek. Administrative, technical, and material support: Mihić and Barać.

\section{References}

1. Millar K, Abildskov J. Notched T waves in young persons with central nervous system lesions. Circulation 37: 597-603, 1968.

2. Van Der Ark GD. Cardiovascular changes with acute subdural hematoma. Surg Neurol 3: 305-312, 1975.
3. Brown RH, Beyerl BD, Iseke R, Lavyne MH. Medulla oblongata edema associated with neurogenic pulmonary edema. J Neurosurg 64: 494-500, 1986.

4. Schroth WS, Tenner SM, Rappaport BA, Mani R. Multiple sclerosis as a cause of atrial fibrillation and electrocardiographic changes. Arch Neurol 49: 422-424, 1992.

5. Uriel N, Kaluski E, Hendler A, Leitman M, Vered Z. Cardiogenic shock in a young female with multiple sclerosis. Resuscitation $\mathbf{7 0}$ : 153-157, 2006.

6. Chagnac Y, Martinovits G, Tadmor R, Goldhammer Y. Paroxysmal atrial fibrillation associated with an attack of multiple sclerosis. Postgrad Med J 62: 385-387, 1986.

7. Crawley F, Saddeh I, Barker S, Katifi A. Acute pulmonary oedema: presenting symptom of multiple sclerosis. Mult Scler 7: 71$72,2001$.

8. Makaryus JN, Kapphahn S, Makaryus AN. Unilateral neurogenic pulmonary oedema and severe left ventricular dysfunction secondary to acute multiple sclerosis exacerbation. Heart Lung Circ 18: 155-158, 2009.

9. Gentiloni N, Schiavino D, Della Corte F, Ricci E, Colosimo C. Neurogenic pulmonary edema: a presenting symptom in multiple sclerosis. Ital J Neurol Sci 13: 435-438, 1992.

10. Drummond JC, Todd MM. Acute sinus arrhythmia during surgery in the fourth ventricle: an indicator of brain-stem irritation. Anesthesiology 60: 232-235, 1984.

11. Kawasaki S, Ishii M, Kon S, Yoshida Y. A case of sporadic and transient bradyarrhythmias in a patient with a glioma in the medulla oblongata. Kokyu To Junkan 41: 787-790, 1993 (in Japanese; abstract in English).

12. Nadkarni T, Kansal R, Goel A. Right cerebellar peduncle neurocysticercosis presenting with bradycardia. Acta Neurochir 152: 731-732, 2010.

13. Netzer F, Bernard JF, Verberne AJ, et al. Brain circuits mediating baroreflex bradycardia inhibition in rats: an anatomical and functional link between the cuneiform nucleus and the periaqueductal grey. J Physiol 589: 2079-2091, 2011.

14. Pelosi GG, Tavares RF, Fernandes KB, Correa FM. Cardiovascular effects of noradrenaline microinjection into the medial part of the superior colliculus of unanesthetized rats. Brain Res 1290: 21-27, 2009.

15. Rocha I, Gonçalves V, Bettencourt MJ, Silva-Carvalho L. Effect of stimulation of sublobule IX-b of the cerebellar vermis on cardiac function. Physiol Res 57: 701-707, 2008.

16. Zadro I, Barun B, Habek M, Brinar VV. Isolated cranial nerve palsies in multiple sclerosis. Clin Neurol Neurosurg 110: 886-888, 
Intern Med 51: 1121-1124, 2012 DOI: 10.2169/internalmedicine.51.7139

2008.

17. Adamec I, Gabelic T, Krbot M, Ozretic D, Milivojevic I, Habek
M. Primary position upbeat nystagmus. J Clin Neurosci 19: 161$162,2012$.

(C) 2012 The Japanese Society of Internal Medicine http://www.naika.or.jp/imindex.html 